01-05

\section{Cannabis use history and onset to psychosis in an ultrahigh-risk group}

\section{RL Greig ${ }^{1,2,3}$, AL Baker ${ }^{2,3}$, MJ Startup ${ }^{1,2,3}$, TJ Lewin ${ }^{3,4}$, SA Halpin ${ }^{2,3}$, VJ Carr ${ }^{3,4}$, J Beckmann ${ }^{2}$}

'School of Behavioural Sciences, The University of Newcastle; ${ }^{2}$ Psychological Assistance Service, Hunter New England Mental Health; ${ }^{3}$ Centre for Mental Health Studies, The University of Newcastle and Hunter New England Mental Health; and ${ }^{4}$ Neuroscience Institute for Schizophrenia and Allied Disorders (NISAD),

New South Wales, Australia

Background: Cannabis is the most widely used illicit drug in Australia with higher rates of use found among those with a mental disorder. The similarities of the effects of cannabis to symptoms of psychosis, particularly schizophrenia, and the observation that cannabis use is temporally linked to the development of schizophrenia symptoms in some people, have led to a plethora of research investigating the relationship between cannabis use and psychosis. Contrary to hypotheses, recent research investigating cannabis use among an 'ultrahigh-risk'-for-psychosis sample found that cannabis use or dependence in the year prior to recruitment to the study was not associated with a heightened risk of developing psychosis over the following 12-month period. The current research was a similar, cross-sectional retrospective study of cannabis use among an ultrahigh-risk group, conducted at the Psychological Assistance Service, Newcastle, New South Wales, from June 2004.

Aims: The study aimed to investigate1) the lifetime patters of cannabis use among an ultrahigh-risk sample; 2) the relationship between cannabis use and risk factors, symptoms and other psychosocial outcomes and 3) the relationship between cannabis use and/or other clinical markers and subsequent transition to psychosis.

Results: Over $75 \%$ of the sample had tried cannabis at service entry and high rates of cannabis use were comparable with other early psychosis cohorts. Earlier cannabis use initiation was associated with increased cannabis use. There was poorer psychosocial functioning in the no-use group and high-use group. High cannabis use alone was not associated with transition to psychosis among the ultrahigh-risk sample; however, findings were in the expected direction. High cannabis use at service entry in combination with family history of psychosis or brief limited psychotic symptoms was associated with significantly greater risk of transition to psychosis.

Conclusions: Cannabis use needs to be addressed in the ultrahigh-risk population. The contribution that cannabis makes to transition to psychosis among an ultrahigh-risk group may be difficult to identify, that is, the proportion of increased risk that cannabis use contributes to developing psychosis may require population studies or larger ultrahigh-risk samples. The neurodevelopmental 'cannabinoid hypothesis' and the 'two-hit' hypothesis of schizophrenia may help guide future research into composite risk factors for psychosis, for example, family history and high cannabis use.

$01-06$

\section{Cognitive behaviour therapy for substance use disorders in people with psychotic disorders}

\author{
A Baker ${ }^{1,2}$, S Bucci ${ }^{3}$, T Lewin $^{1,3}$, F Kay-Lambkin', \\ P Constable ${ }^{3}$, V Carr ${ }^{1,2}$
}

'Neuroscience Institute of Schizophrenia and Allied Disorders (NISAD), New South Wales, Australia; ${ }^{2}$ The University of Newcastle, Callaghan, New South Wales, Australia; ${ }^{3}$ Hunter New England Health, Newcastle, Australia

Background: Despite the widespread co-occurrence of psychosis and substance use disorders and the adverse effects of substance use on functioning and outcome among people with psychosis, few randomized controlled trials specifically aimed at reducing substance use among people with psychotic disorders have been conducted.

Aim: The aim of the study was to investigate whether a 10 -session motivational interviewing and cognitive behavioural therapy (MI/CBT) intervention among a sample of people with psychosis and substance use disorders was more efficacious than routine treatment in reducing substance use and improving symptomatology and general functioning.

Methods: Participants were a community sample who met a clinical diagnosis for a psychotic illness in accordance with the Diagnostic Interview for Psychosis and reported hazardous alcohol, cannabis and/or amphetamine use on the Opiate Treatment Index during the past month. Participants were randomly allocated to receive 10 sessions of MI and CBT $(n=65)$ or treatment as usual $(n=65)$ and were assessed on multiple outcomes at posttreatment, 6 and 12 months after pretreatment.

Results: There was a short-term improvement in cannabis use and depression among those receiving the $\mathrm{MI} / \mathrm{CBT}$ intervention, together with impacts on general functioning at 12 months. There was no differential benefit of the intervention on substance use at 12 months, except for a potentially clinically important effect on amphetamine use. Assessment and brief advice in the context of ongoing monitoring 
appeared to have an overall beneficial effect on alcohol consumption.

Conclusions: Further research is needed to evaluate the specific impacts on regular amphetamine use and to develop more efficacious interventions among regular cannabis users. A stepped-care approach to interventions for excessive alcohol consumption among people with a psychotic disorder is recommended.

01-07

\section{Cannabis use as a complication in psychological treatments for people at ultrahigh risk of psychosis: early findings from the DEPTh trial}

\section{Startup ${ }^{1,2}$, V Carr ${ }^{2,3}$, A Baker ${ }^{2,3}$, H Stain ${ }^{3,4}$, S Halpin', S Bucci ${ }^{5}$}

${ }^{1}$ School of Psychology, The University of Newcastle, Australia; ${ }^{2}$ Centre for Mental Health Studies, The University of Newcastle; ${ }^{3}$ Neuroscience Institute of Schizophrenia and Allied Disorders (NISAD), New South Wales, Australia; ${ }^{4}$ Centre for Rural \& Remote Mental Health, The University of Newcastle; ${ }^{5}$ sychological

Assistance Service, Hunter New England Area Health Service, Newcastle, Australia

The DEPTh project is a randomized controlled trial comparing the effectiveness of cognitive behaviour therapy (CBT) and person-centered therapy for the prevention of psychosis among young people who are at ultrahigh risk. It is being conducted on two sites in New South Wales: the Hunter Valley and the Greater Western Area. The CBT is modeled on the manual developed by Paul French and Tony Morrison in the UK. This form of CBT has been shown in one trial to be effective in preventing transition to psychosis. However, it does not include any techniques that specifically focus on the reduction of substance abuse even though such abuse, especially of cannabis, is known to be very high among people who are at ultrahigh risk, and cannabis use has been found in prospective studies to be associated with the later onset of psychosis. Therefore, we have adapted French and Morrison's CBT by integrating it with a manual for motivational interviewing (MI) with young cannabis users. MI is directed toward the resolution of ambivalence and movement toward change, with the resources and motivation to change considered to reside within the client. The therapist's task is to create a set of conditions that will enhance the client's own motivation and commitment for change by drawing on their personal perceptions, goals and values. The ways in which CBT and MI are integrated in the DEPTh project will be illustrated with case material.

\section{Personality Disorders (International Society for the Study of Personality Disorders Symposium)}

\author{
AM Chanen \\ ORYGEN Research Centre, Parkville, Victoria, Australia
}

\section{Overview}

This symposium brings together researchers from Australia and New Zealand affiliated with the International Society for the Study of Personality Disorders. It canvasses current personality disorder research from neurobiology through to interpersonal processes and intervention across the life span from youth to old age.

02-01

\section{Emotion processing and regulation in first-presentation borderline personality disorder}

\section{NB Allen, S Chong, AM Chanen}

ORYGEN Research Centre and Department of Psychology, The University of Melbourne, Melbourne, Australia

Emotion dysregulation is widely believed to be one of the core features of borderline personality disorder (BPD). To date, there has been no study examining psychophysiological and self-report measures of deliberate regulation of emotions in BPD in those in the early phases of the disorder. The aims of the study were to measure the 1) psychophysiological and subjective emotional response and 2) psychophysiological and subjective ability to regulate one's emotional response to emotional images in 15- to 24-year-olds with first-presentation BPD compared with healthy controls. Twenty patients with full-syndrome BPD and 20 healthy comparison participants were examined. Test stimuli used were a set of standardized photographic images with pleasant, neutral or unpleasant valence. Participants were also instructed to either 'maintain' or 'suppress' the emotional response they were having to the stimuli. In addition to self-report ratings, emotional responses to the stimuli were measured by startle response and skin conductance. Contrary to the hypotheses, self-report and psychophysiological data did not provide evidence that the BPD participants reacted with more intense affective responses 